\title{
Force Modulation Microscopy (FMM) of Styrene Tri-block Copolymer with Styrene Maleic-Anhydride
}

\author{
Bongwoo Kang, Lan Pham and Changmo Sung \\ Department of Chemical and Nuclear Engineering, Center for Advanced Materials \\ University of Massachusetts, Lowell, MA 01854
}

Polymer blending has been a technique used in past decades to create new types of polymers with desirable properties. SIBS can be applied to drug delivery system and through blending with SMA, mechanical property will be improved. Classically to identify two phase polymer samples, TEM, SEM or AFM topography have been used, but methods were not reliable. We have tried Force Modulation Microscopy (FMM) to characterize two phases in the blended polymer without staining.

This study examined the morphology of SIBS and SMA blends at different compositions by FMM as the two polymers have different elastic properties and these images were compared with Scanning Electron Microscopy (SEM) images. To distinguish the two phases by FMM, samples were embedded in epoxy and polished to produce a smooth flat surface. FMM images were obtained using a PSIA XE-100. A non-contact tip, stiffer than contact tip, with on silicon cantilever was used. The cantilever's length and width was $125 \mu \mathrm{m}$ and $35 \mu \mathrm{m}$, respectively. The cantilever had a resonant frequency of $325 \mathrm{kHz}$ and a force constant of $40 \mathrm{~N} / \mathrm{m}$. Choosing the cantilever is a very critical factor in FMM. Phase and Force Modulation images were obtained at optimized scanning conditions. Image analysis has been carried to measure volume fraction from FMM images at different blending ratios.

The microstructure depended on the composition of the blend, compatibility of the two components, fabrication of the blended materials, and the physical properties of the two polymers. Fig. 1 shows the presence of the two phases clearly with corresponding physical properties. The brighter contrast shows SMA phase, hard segment, and the darker contrast reveals SIBS phase, soft segment. From the image analysis by GAIA Blue software with FMM image, volume fraction of SMA was calculated as 18.8\% Fig. 1(a), 42.5\% Fig. 1(b) and 23.0\% Fig. 1(c). These values are in good agreement with blending ratio used to formulate the blends. FMM studies of SIBS/PS system were also carried out. FMM images revealed differences two phases clearly, even though SEM did not show any phase separation images due to poor contrast of two phases. In a conclusion, FMM is proved to be unique and powerful method to characterize phase separation of polymer blend.

Reference

[1] D. R. Paul and S. Newman (eds.), "Plymer Blends, Vol. 1 and 2," Academic Press, New York, 1978.

[2] D. R. Paul, Ch. 12 in "Polymer Blends, Vol. 2," D. R. Paul and S. Newman (eds.), Academic Press, New York, 1978.

[3] N. Dharmarajan and S. Datta, Polymer, 33, 3848 (1992).

[4] S. Datta, N. Dharmarajan, G. Verstrate, and L. Ban, Polym. Eng. Sci., 33, 721 (1993).

[5] J.H. Kim, H. Keskkula, and D. R. Paul, J. Appl. Polym. Sci., 40, 183 (1990). 
(a)

(b)
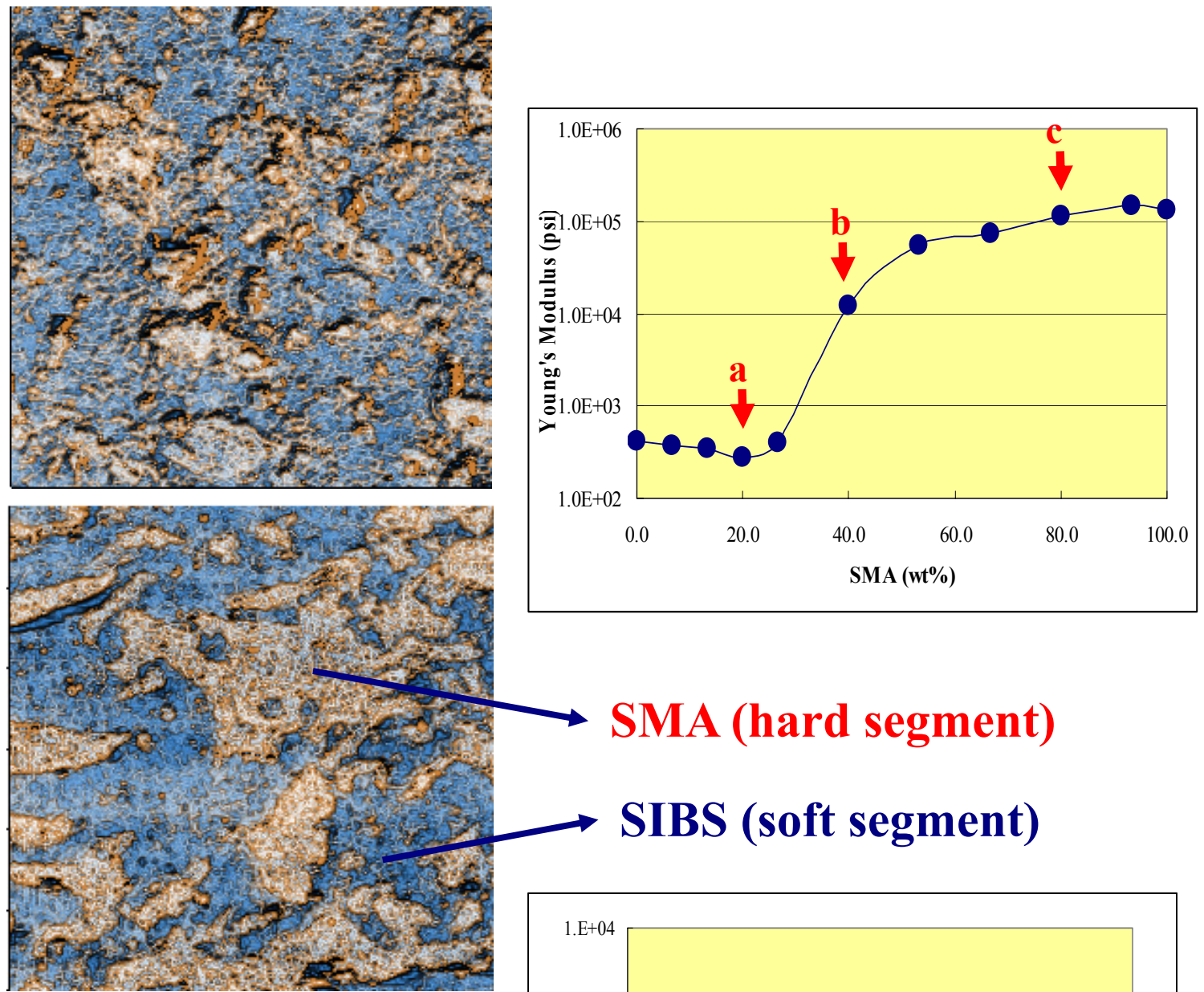

(c)
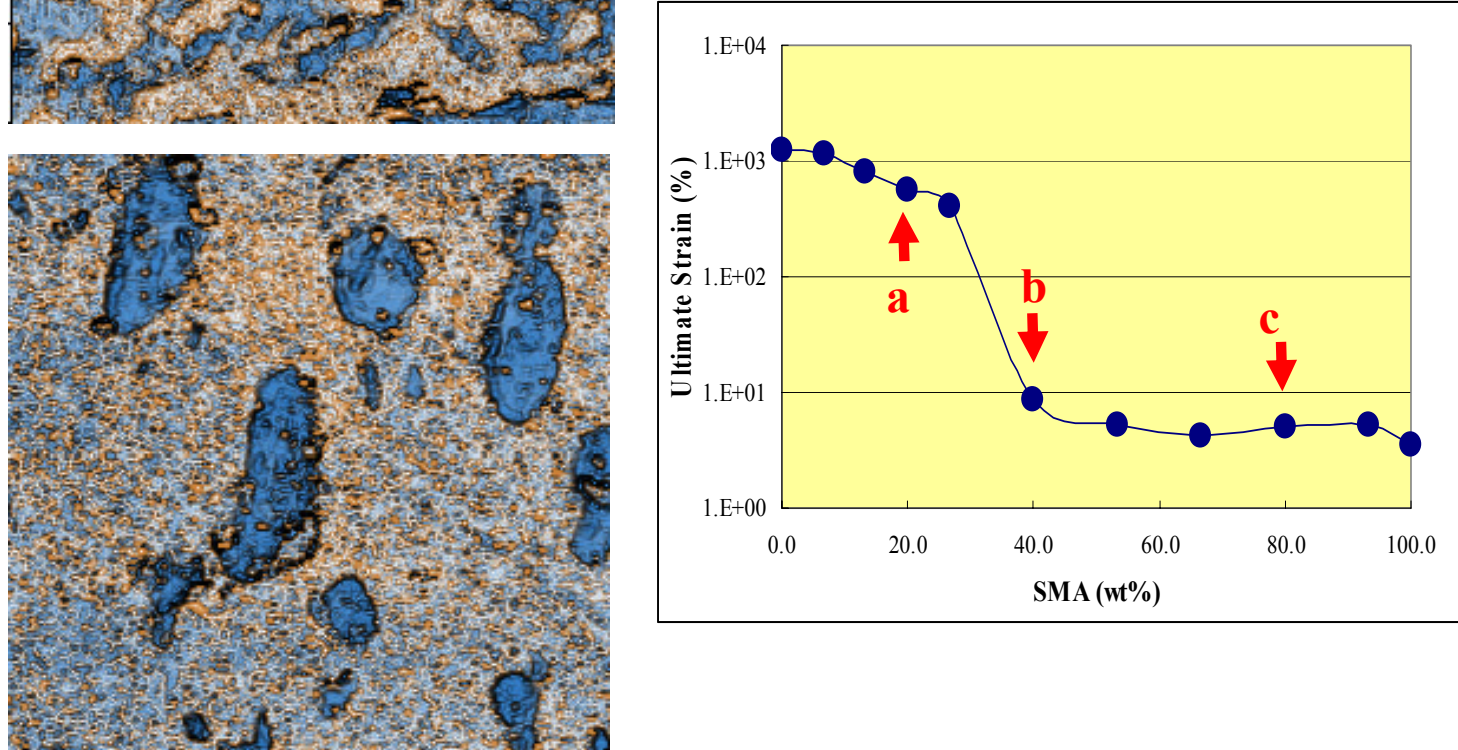

FIG. 1. Mechanical properties and FMM images at 30 $\mu \mathrm{m}$ x $30 \mu \mathrm{m}$ scan size. (a) $20 \mathrm{wt} \% \mathrm{SMA} / 80 \mathrm{wt} \%$ SIBS1027, (b) 40wt\% SMA/60wt\% SIBS1027, and (c) 80wt\% SMA/20wt\% SIBS1027. 